\title{
Successful Antegrade Recanalization of Chronic Total Aorto-ostial in Stent Occlusion of Right Coronary Artery with Previously Implanted Protruding Stent
}

\author{
Zivile Valuckiene $^{1 *}$ and Aigars Lismanis ${ }^{2}$ \\ Department of Cardiology, Medical Academy, Lithuanian University of Health Sciences, Kaunas, Lithuania
}

Latvian Centre of Cardiology, Pauls Stradins Clinical University Hospital, Riga, Latvia

${ }^{*}$ Corresponding author: Zivile Valuckiene, Department of Cardiology, Hospital of Lithuanian University of Health Sciences Kaunas Clinics, Eiveniu g. 2, LT-50009 Kaunas, Lithuania, Tel: +370 641 78145; Fax: (+370 37) 3313 95, E-mail: z.valuckiene@gmail.com

Rec. Date: Aug 25, 2015; Acc. Date: Sep 25, 2015; Pub. Date: Sep 29, 2015

Copyright: ( 2015 Valuckiene Z, et al. This is an open-access article distributed under the terms of the Creative Commons Attribution License, which permits unrestricted use, distribution, and reproduction in any medium, provided the original author and source are credited.

\begin{abstract}
Recanalization of coronary artery chronic total occlusions is a rapidly developing domain of percutaneous coronary interventions requiring considerable operator expertise. Tackling any aorto-ostial lesion with previously implanted stent that protrudes into aorta presents anatomical and technical challenge significantly reducing procedure success rates and is an obstacle when considering antegrade approach in chronic total occlusion cases. We present an illustrative case report of successful antegrade recanalization of chronic total aorto-ostial in-stent occlusion of right coronary artery with previously implanted protruding stent.
\end{abstract}

Keywords: Chronic total occlusion; Antegrade recanalization; Percutaneous coronary intervention; Aorto-ostial protruding stent

\section{Introduction}

Chronic Total Occlusion (CTO) recanalization represents the summit of advance in contemporary Percutaneous Coronary Intervention (PCI) techniques and operator expertise. Aorto-ostial lesions with previously implanted stents protruding into aorta are technically challenging even in non-CTO cases. They are known to significantly reduce procedure success rates and present a considerable drawback from attempting antegrade approach in CTO PCI. Protrusion of aorto-ostial stents accounts for suboptimal or difficult guiding catheter engagement, lack of support and wire control, difficulties in delivery of micro catheters, balloons and stent systems [1]. This case report illustrates successful antegrade recanalization of chronic total aorto-ostial in-stent occlusion of the right coronary artery (RCA) with previously implanted protruding stent.

\section{Case Report}

Fifty eight years old man presented with long-standing history of class 3 stable angina in the year 2014, and history of previous non-Q wave myocardial infarction in 2004 treated by PCI to the left Circumflex Coronary Artery (LCx) and proximal to mid RCA stenting with 2 drug eluting stents (total stented segment length 52 millimetres) in the year 2009. Resting ECG was unremarkable, and cardiac sonography revealed good regional left ventricular function with preserved ejection fraction. The exercise stress test was positive with ischemic ECG changes in inferior leads. Subsequently the patient underwent coronary angiography, which showed mild diffuse atherosclerosis in the Left Coronary Artery (LCA) with unobstructed LCx stents and a long (52 millimetres) in-stent RCA occlusion from the ostium to mid segments of the vessel with distal segments filling retrogradely from collaterals supplied by LCA. Percutaneous coronary intervention to RCA with antegrade approach has been attempted and abandoned by the first operator with reported inability to pass the wire into the true lumen of the vessel. Four months later due to persisting symptoms despite optimal medical treatment the patient has been referred to high-volume interventional cardiologist with special interest in CTO PCI for repeated RCA recanalization attempt. Bilateral femoral approach with $35 \mathrm{~cm}$ 7F sheath for guiding catheter insertion and $5 \mathrm{~F}$ sheath for contralateral contrast injection has been selected. Left Amplatz (AL) guiding (7F, sizes 0.75, 1, 1.5), and diagnostic Judkins right catheters were used to selectively intubate the ostium of RCA with no initial success. Suboptimal AL 0.75 guiding catheter position above the ostium of RCA (Figure 1A) with contrast injection has showed a small blunt aorto-ostial occlusion stump with no antegrade flow (TIMI 0) and protrusion of previously implanted RCA stent of approximately 4 millimetres into the aorta explaining the difficulty in selective ostial RCA intubation (Figure 1B).

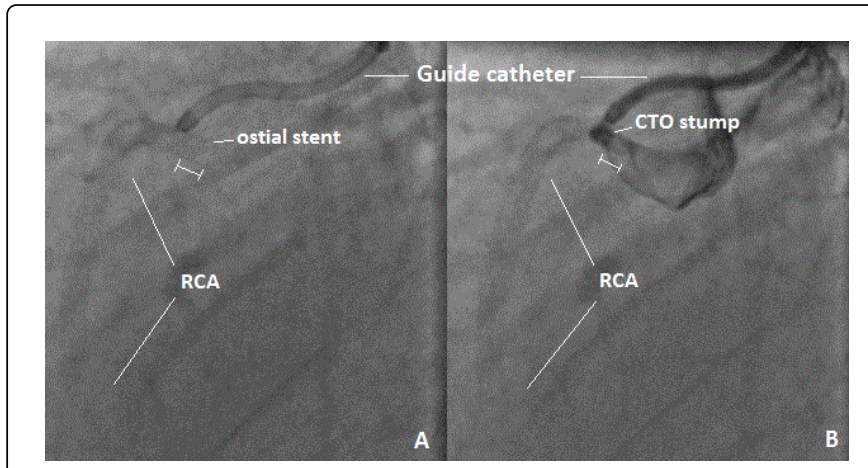

Figure 1: (LAO 90o) A. Amplatz left 0.75 7F guiding catheter positioned above the ostium of right coronary artery (RCA) with visible aorto-ostial stent protrusion into aorta $(|-|$ marker). B. Antegrade contrast injection showing blunt aorto-ostial RCA stump with no antegrade flow (TIMI 0).

After repeated numerous attempts to intubate the ostial RCA stump, coaxial guiding catheter position was finally achieved. Contralateral contrast injection allowed visualisation of distal RCA segments filling 
Citation: Valuckiene Z, Lismanis A (2015) Successful Antegrade Recanalization of Chronic Total Aorto-ostial in Stent Occlusion of Right Coronary Artery with Previously Implanted Protruding Stent. J Vasc Med Surg 3: 223. doi:10.4172/2329-6925.1000223

Page 2 of 4

retrogradely from collateral supply and estimation of CTO length (Figure 2).

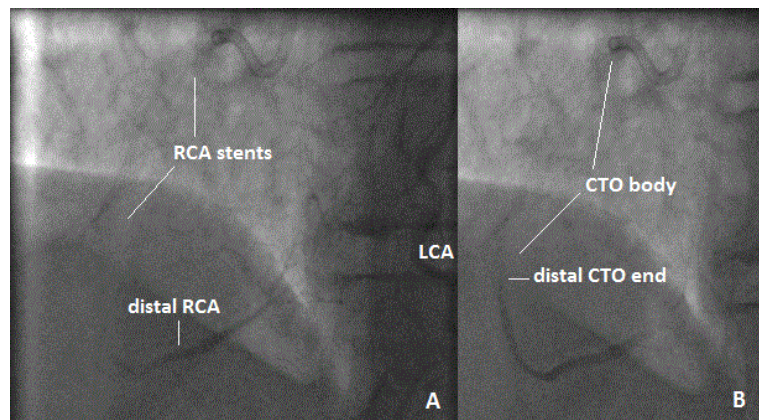

Figure 2: (LAO 30o CRAN 13o) A. Retrograde contrast injection from left coronary artery (LCA) depicting weak thread-like collaterals (Rentrop 2) to distal right coronary artery (RCA) segments. B. Late retrograde filling phase showing distal end of chronic total occlusion (CTO) of RCA allowing estimation of the length of the lesion (CTO body).

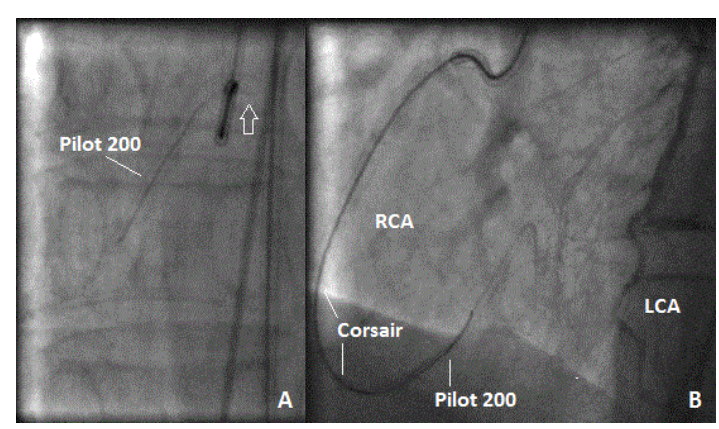

Figure 3: A (AP) Pilot 200 guide wire crossing in-stent chronic total occlusion (CTO) of the right coronary artery (RCA). Protrusion of previously implanted aorto-ostial stent is visible to the right side of the guiding catheter, which is disengaged from the ostium with looping wire (arrow) while its tip is negotiating the path of CTO lesion. B (LAO 33o CRAN 11o). Retrograde contrast injection from the left coronary artery (LCA) confirming distal Pilot 200 guide wire position in the true lumen of RCA followed by Corsair micro catheter for further guide wire exchange.

Step up in wire stiffness from Asahi Soft to Gaia First and then to Pilot 200 was needed to cross the CTO. It was technically difficult to maintain stable guiding catheter position throughout the procedure as catheter was backing out while wire was crossing the occlusion site (Figure 3A). Micro catheter Corsair has been used for wire back up and transit exchange (Figure 3B). After crossing the CTO lesion with Pilot 200 wire Asahi Sion Blue wire was positioned distally in RCA via Corsair micro catheter in order to reduce risk of distal vessel perforation, and subsequent balloon angioplasty with compliant $2 \times 30$ $\mathrm{mm}$ and $2.5 \times 40 \mathrm{~mm}$ balloons was performed within the occluded segments resulting in restoration of antegrade TIMI 3 flow in the artery. However, RCA ostium remained significantly stenosed despite compliant balloon inflation. Consequently, decision to use a larger Non-Compliant (NC) balloon has been made. When trying to deliver a
$3.5 \times 30 \mathrm{~mm}$ NC balloon into the vessel for additional ostial dilatation the guiding catheter prolapsed into aorta pulling the wire and the balloon out of the artery. Selective ostial recatheterization with AL catheter again has proven to be extremely difficult and consequently catheter was changed to right coronary bypass guide, which appeared engaged from standard Left Anterior Oblique (LAO) projection.

The artery was rewired and subsequent balloon angioplasty with $3.5 \times 30 \mathrm{~mm}$ NC balloon (20 Atm) was carried out. Angiographic cine loop showed ostial RCA stent crushed sideways by inflated balloon (Figure 4A). The final angiographic result was acceptable (Figure 4B and $4 \mathrm{C}$ ), therefore no additional stent implantation has been performed at the time. Subsequent frame-by-frame analysis has revealed that RCA has been rewired through the stent side-struts after losing guidewire and catheter position, and this important finding was omitted at misleading LAO view (Figure 5). No procedure-related or post-procedural complications have been observed. The patient continued on regular medical treatment with dual antiplatelet therapy and anginal symptoms resolved.

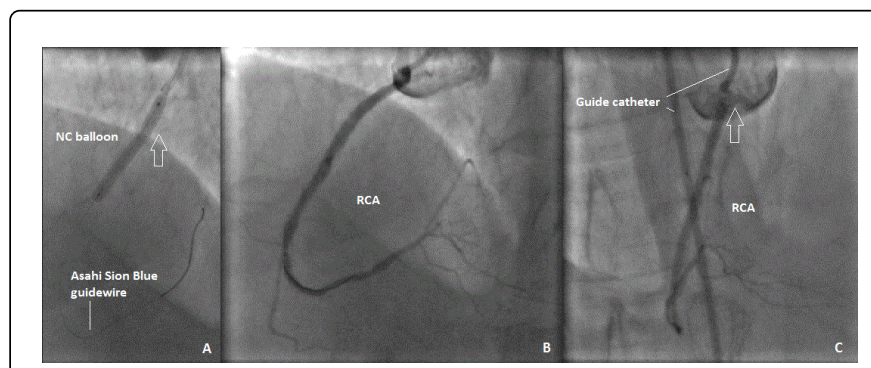

Figure 4: A (LAO 28o CRAN 25o). Non-compliant (NC) 3.5x30 $\mathrm{mm}$ balloon inflated at $18 \mathrm{Atm}$ in aorta and proximal part of right coronary artery (RCA) opening aorto-coronary side struts of previously implanted RCA stent. Aortic stent protrusion is illustrated with an arrowhead. B (LAO 28o CRAN 25o). Final result after RCA CTO recanalization and balloon angioplasty with antegrade TIMI 3 flow in the artery. C (RAO 9o CRAN 27o). Cranial view with slight right anterior oblique tilt illustrates final result after guide wire removal with patent RCA ostium and deformed segment of proximal RCA stent in the aorta (arrowhead).

\section{Discussion}

This case report presents a challenging CTO lesion scenario with ample negative predictors of percutaneous recanalization success: occluded segment of $>20 \mathrm{~mm}$ length, short aorto-ostial blunt stump, impeded ostial access by previously implanted RCA stent protruding into aorta, and previously failed antegrade PCI attempt (J-CTO score $=3$ ) [2]. However, as proven by contemporary international registries these factors should not discourage another attempt by experienced operators as high success rates are still reported in the same or different operator's hands. Careful beforehand procedure planning is essential to achieve maximal success rates and expert consensus is that CTO PCI should never be performed ad hoc or under time limit constraints [3]. Adequate back-up to allow wire crossing the CTO lesion is a technical cornerstone in CTO PCI which starts with arterial access site selection. Lower procedure success rates are reported in the literature with radial compared to femoral access even despite lower lesion complexity in the radial subgroup [4]. Femoral access is often superior to radial arterial access in CTO PCI in 
providing maximal options for catheter size selection with possibility to add extra back up with longer introducer sheaths. The lack of guiding catheter support often results in difficulty to control and manipulate technical equipment during the procedure and may be the primary cause of procedural failure. Therefore, at least 7F arterial sheath and guiding catheters are usually needed for complex CTO PCI cases to accommodate multiple wires, micro-catheters, balloons or other devices required to pursue and complete the procedure. Simultaneous antegrade and retrograde contrast injections is the standard technique to estimate CTO length, anatomical features and distal artery segments in the absence of antegrade filling from ipsilateral/bridging collaterals or intra-CTO micro channels as well as to confirm intraluminal wire position in the vessel [3]. Generally, softcurved (Judkins type) guiding catheters with side holes are preferred for ostial RCA lesions due to often deeper arterial intubation, pressure dampening, plaque burden and spastic component, as well as it holds important preventive role in causing or extending already present iatrogenic coronary artery dissections by facilitating early contrast exit with antegrade injections. However, in this case of ostial RCA CTO with minimal stump, difficult ostial catheterization and no possibility to employ anchoring techniques left Amplatz catheter (sizes 0.75-2) was the only option.

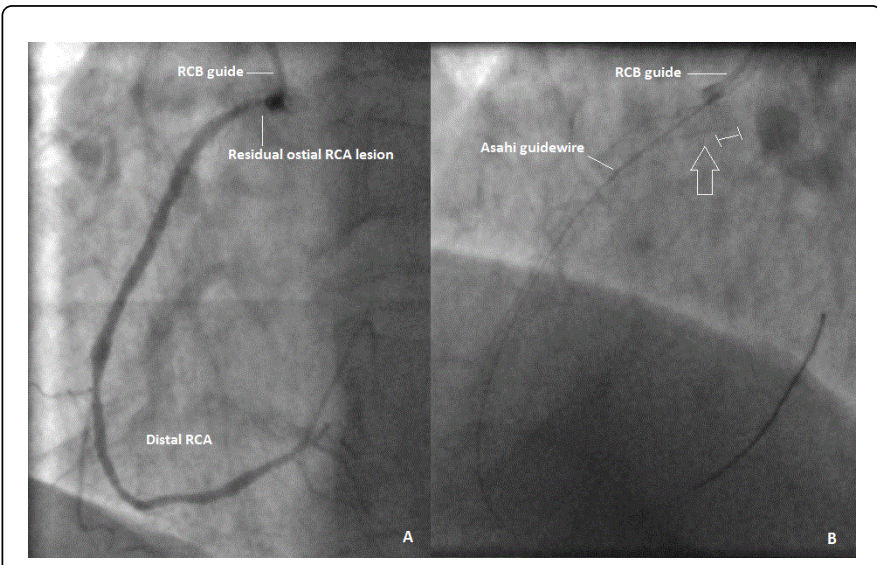

Figure 5: A (LAO 23o) Residual ostial right coronary artery (RCA) lesion after balloon angioplasty with compliant balloons. Position of right coronary bypass $(\mathrm{RCB})$ guiding catheter appears engaged to the ostium of the artery at this view. B (LAO 33o CRAN 18o). Frozen cine loop frame showing superior malposition of the guiding catheter and guide wire entering RCA lumen through the side struts of ostially protruding stent (arrowhead shows real ostium of RCA and $|-|$ marker represents protruding segment of the stent).

Better understanding of the histopathology of CTO lesions allows selection of the 'right' wires, which may facilitate the lesion crossing. Routinely the CTO operator starts probing the lesion with the soft wire aiming for microchannels or soft tissue tracking along the CTO length, which are often present irrespectively of CTO duration or length and angiographic appearance [5]. However, proximal and distal fibrous caps are often difficult to penetrate with soft wires only. Since in-stent restenosis (even its long-standing occlusive form) is almost exclusively the result of neointimal hyperplasia, after trying first-choice soft wires step up to hydrophilic polymer coated Pilot 200 wire was made, which turned out to success in our case.
The biggest obstacle in this procedure was protrusion of ostial RCA stent, which rendered guiding catheter unstable and difficult to reengage upon wire and device manoeuvres despite maximal back up from femoral access site, extra-long introducer sheath and aggressive 7F AL 0.75 curve. Different operators have suggested ways to achieve coaxial guiding catheter position in challenging scenarios of protruding ostial stents by using a double wire technique [6] or circumvent the need of co-axial guiding catheter position by entering the vessel lumen through or under the struts of protruding stent [7-9]. All these options have been employed in the setting of critical or subocclusive in-stent disease with present antegrade flow and some patency of the vessel lumen. However, such approaches have never been described in CTO cases. Rewiring the artery through the stent side-struts in our case has occurred as a non-intentional event after losing the guide catheter and wire position and misinterpreting the guiding catheter as engaged coaxially at isolated standard LAO view. This unwanted event could have been avoided by double-checking the guiding catheter and wire position at LAO view with cranial tilt.

All aorto-ostial PCIs are complex as require precise ostial coverage without excessive protrusion of the stent struts in the aorta. A study performed by Dishmon et al. has shown that in ostial lesion PCI cases stents are implanted too proximally in at least half of PCI procedures guided by conventional visual angiography, resulting in inability to repeatedly catheterize the ostium in more than $90 \%$ of such situations [1]. However angiographically guided ostial stenting is still predominant approach in aorto-ostial lesions PCIs of many operators in daily practice. Various techniques have been created to aid operators in defining the exact ostial landing zone of the proximal part of the stent, however none of them is proven to be superior or universally effective [10].

The best treatment for patients with drug eluting stent restenosis is still debatable. Different investigators suggest drug eluting balloon angioplasty or trying a new drug eluting stent during the repeated interventions, however long-term restenosis rates are still high [11]. Side-strut crossing and stenting techniques for aorto-ostial in-stent restenosis have been described as options to approach this type of lesions, however such reports are not numerous and definite long term follow up results are unavailable [7-9]. We chose to carry out plain high-pressure non-compliant balloon angioplasty alone, which allowed maximal opening of the ostial RCA with no angiographically visible neointimal or stent strut recoil, and further clinical follow up strategy.

Retrograde approach in CTO recanalization is increasingly used with high success rates and has even been reported as primary approach method in estimated difficult antegrade access cases by various operators [12-14]. Reports of retrograde delivery of stent to RCA via septal perforators in cases of challenging antegrade RCA ostial approach are also present [15]. Retrograde recanalization approach would have been an option in this case if antegrade attempt to recanalize CTO had failed.

\section{Conclusion}

CTO interventions are generally challenging and demanding, and carry potential pitfalls in all stages of revascularization procedure. The clues to success are operator persistence, confidence in handling contemporary CTO PCI tools and techniques, and ability to select appropriate management strategy in various procedure scenarios. 
Citation: Valuckiene Z, Lismanis A (2015) Successful Antegrade Recanalization of Chronic Total Aorto-ostial in Stent Occlusion of Right Coronary Artery with Previously Implanted Protruding Stent. J Vasc Med Surg 3: 223. doi:10.4172/2329-6925.1000223

Page 4 of 4

\section{References}

1. Dishmon DA, Elhaddi A, Packard K, Gupta V, Fischell TA (2011) High incidence of inaccurate stent placement in the treatment of coronary aorto-ostial disease. J Invasive Cardiol 23: 322-326.

2. Morino Y, Abe M, Morimoto T, Kimura T, Hayashi Y, et al. (2011) Predicting successful guidewire crossing through chronic total occlusion of native coronary lesions within 30 minutes: the J-CTO (Multicenter CTO Registry in Japan) score as a difficulty grading and time assessment tool. JACC Cardiovasc Interv 4: 213-221.

3. Karmpaliotis D, Stone WG (2014) Percutaneous recanalisation of coronary artery chronic total occlusions: a 2014 update. EMJ Int Cardiol 1:28-37.

4. Burzotta F, De Vita M, Lefevre T, Tommasino A, Louvard Y, et al. (2014) Radial approach for percutaneous coronary interventions on chronic total occlusions: technical issues and data review. Catheter Cardiovasc Interv 83: 47-57.

5. Sumitsuji S, Inoue K, Ochiai M, Tsuchikane E, Ikeno F (2011) Fundamental wire technique and current standard strategy of percutaneous intervention for chronic total occlusion with histopathological insights. JACC Cardiovasc Interv 4: 941-951.

6. Chetcuti SJ, Moscucci M (2004) Double-wire technique for access into a protruding aorto-ostial stent for treatment of in-stent restenosis. Catheter Cardiovasc Interv 62: 214-217.

7. Burstein JM, Hong T, Cheema AN (2006) Side-strut stenting technique for the treatment of aorto-ostial in-stent restenosis and deformed stent struts. J Invasive Cardiol 18: E234-237.
8. Kaluski E, Hendler A, Klapholz M (2007) PCI of the right coronary artery via or under struts of stents protruding into the aorta. J Invasive Cardiol 19: E207-209.

9. Kaplan S, Barlis P, Tanigawa J, Goktekin O, Di Mario C (2008) Unconventional treatment of aorto-ostial instent restenosis with marked protrusion into the aorta. J Cardiovasc Med (Hagerstown) 9: 184-186.

10. Kwan TW, James D, Huang Y, Liou M, Wong S, et al. (2012) Perfection of precise ostial stent placement. J Invasive Cardiol 24: 354-358.

11. Fernando A (2009) Treatment of In-stent Restenosis - Past, Present and Future. European Cardiology Review 2: 74-78.

12. Londoño JC, Singh V, O'Neill WW (2011) Total aorto-ostial chronic occlusion of the right coronary artery successfully recanalized via retrograde approach. Catheter Cardiovasc Interv 78: 400-404.

13. Sianos G, Barlis P, Di Mario C, Papafaklis MI, Büttner J, et al. (2008) European experience with the retrograde approach for the recanalisation of coronary artery chronic total occlusions. A report on behalf of the euroCTO club. EuroIntervention 4: 84-92.

14. Galassi AR, Tomasello SD, Reifart N, Werner GS, Sianos G, et al. (2011) In-hospital outcomes of percutaneous coronary intervention in patients with chronic total occlusion: insights from the ERCTO (European Registry of Chronic Total Occlusion) registry. EuroIntervention 7: $472-479$.

15. Utunomiya M, Katoh O, Nakamura S (2009) Percutaneous coronary intervention for a right coronary artery stent occlusion using retrograde delivery of a sirolimus-eluting stent via a septal perforator. Catheter Cardiovasc Interv 73: 475-480. 